\title{
Retinal blood vessel segmentation from retinal image using B-COSFIRE and adaptive thresholding
}

\author{
Aziah Ali', Wan Mimi Diyana Wan Zaki', Aini Hussain ${ }^{3}$ \\ ${ }^{1,2,3}$ Center for Integrated Systems Engineering and Advanced Technologies (INTEGRA), \\ Faculty of Engineering \& Built Environment, Universiti Kebangsaan Malaysia, Malaysia \\ ${ }^{1}$ Faculty of Computing \& Informatics, Multimedia University, Malaysia
}

\section{Article Info}

Article history:

Received Sep 15, 2018

Revised Nov 21, 2018

Accepted Dec 2, 2018

\section{Keywords:}

Adaptive thresholding

B-COSFIRE filter

Retinal blood vessel

Segmentation

\begin{abstract}
Segmentation of blood vessels (BVs) from retinal image is one of the important steps in developing a computer-assisted retinal diagnosis system and has been widely researched especially for implementing automatic BV segmentation methods. This paper proposes an improvement to an existing retinal BV (RBV) segmentation method by combining the trainable BCOSFIRE filter with adaptive thresholding methods. The proposed method can automatically configure its selectivity given a prototype pattern to be detected. Its segmentation performance is comparable to many published methods with the advantage of robustness against noise on retinal background. Instead of using grid search to find the optimal threshold value for a whole dataset, adaptive thresholding (AT) is used to determine the threshold for each retinal image. Two AT methods investigated in this study were ISODATA and Otsu's method. The proposed method was validated using 40 images from two benchmark datasets for retinal BV segmentation validation, namely DRIVE and STARE. The validation results indicated that the segmentation performance of the proposed unsupervised method is comparable to the original B-COSFIRE method and other published methods, without requiring the availability of ground truth data for new dataset. The Sensitivity and Specificity values achieved for DRIVE and STARE are $0.7818,0.9688,0.7957$ and 0.9648 , respectively.
\end{abstract}

Copyright @ 2019 Institute of Advanced Engineering and Science. All rights reserved.

\section{Corresponding Author:}

Aini Hussain,

Center for Integrated Systems Engineering and Advanced Technologies (INTEGRA),

Faculty of Engineering \& Built Environment,

Universiti Kebangsaan Malaysia, 43600 Bangi, Malaysia.

Email: draini@ukm.edu.my

\section{INTRODUCTION}

It is projected that diabetes cases worldwide will see a steady rise in the near future which in turn will increase the cases of diabetic retinopathy (DR). DR is an ocular disease affecting diabetic patients which could lead to total blindness if the patients are not given timely treatment. To decrease the risk of total blindness, many hospitals worldwide are now monitoring diabetic patients for any DR symptoms via routine retinal screening. This results in a large number of retinal images to be diagnosed by ophthalmologists. A computer-assisted retinal diagnosis system can be developed to assist the ophthalmologists in performing a more efficient and accurate retinal diagnosis when dealing with large number of retinal images.

One of the crucial steps in a computer-assisted retinal diagnosis system is accurate detection of RBVs from a retinal image, or referred to as RBV segmentation. By analysing the RBV structures produced by segmentation algorithm, ophthalmologists can detect and diagnose a number of ocular diseases such as Diabetic Retinopathy, macular degeneration and glaucoma. Techniques for automatic segmentation of RBVs have been a major research topic in retinal image processing field. Reviews of current techniques for this 
purpose have been comprehensively summarised by Fraz [1] and recently by Almotiri [2]. These techniques can be generally divided into two main categories, namely supervised and unsupervised methods. Supervised methods require manually segmented RBVs image to be available for training models to be used later to classify pixels. Unsupervised methods on the other hand does not need to have a prior training session, but use rule-based methods to identify vessel pixels against background pixels. Some of the available techniques include kernel-based method [3]-[5], vessel tracking method [6], [7], mathematical morphologybased method [8], [9], multi-scale method [10]-[12], machine learning method [13]-[16] and model-based method [17], [18].

B-COSFIRE, stands for Bar-selective Combination of Shifted Filter Responses, is an efficient method to segment RBV from digital fundus images [19]. The resulting segmented vessel tree (VT) with BCOSFIRE for both DRIVE and STARE datasets demonstrated the method's capability to accurately detect main VT on fundus images. The method is also robust against the noise from non-vessel structures on the retinal image background compared to other existing segmentation methods. Of all the published methods so far, B-COSFIRE method demonstrated its superiority in the efficiency of segmenting RBVs from a retinal image compared to other published techniques [19]. The method only takes approximately six seconds on average to produce the final segmented vessel output image with images from DRIVE and STARE. This lends the method well to real-time applications of BV segmentation in retinal image diagnosis system. The method could also be tailored to suit mobile computing in the future which requires low computational load and short processing time. While the method has a relatively large number of parameters to be determined for each dataset to process, this can be resolved by using the parameter estimation method proposed by Vostatek in [20].

In this study, the effectiveness of using adaptive thresholding with B-COSFIRE filter to binarize the grayscale image was investigated. Threshold value was originally determined using grid search by Azzopardi [19], which requires availability of ground truth image. The threshold value was systematically adjusted and optimal value was selected as the value that maximized a chosen segmentation performance metric called MCC for all the images in a dataset. By using adaptive thresholding, we can eliminate the need to empirically determine a threshold value for each new dataset to process which requires ground truth data. Two adaptive thresholding methods were investigated in this study, namely ISODATA and Otsu's method. The following Section 2 describes the background of B-COSFIRE method and how it performs RBV detection on retinal image. The mechanism of the two adaptive thresholding methods are also described in Section 2 followed by the overview of the proposed method in combining adaptive thresholding with B-COSFIRE method. Experimental results of the proposed method validated on DRIVE and STARE dataset are presented in Section 3 and the conclusion in Section 4.

\section{RESEARCH METHOD}

\subsection{Overview}

A number of pre-processing steps are applied to the original color retinal image before B-COSFIRE filter is applied to obtain the vessel-enhanced gray image output. In order to produce the final binary image of the VT, adaptive thresholding is applied on B-COSFIRE output image. An overview of the proposed method is illustrated in the Figure 1.

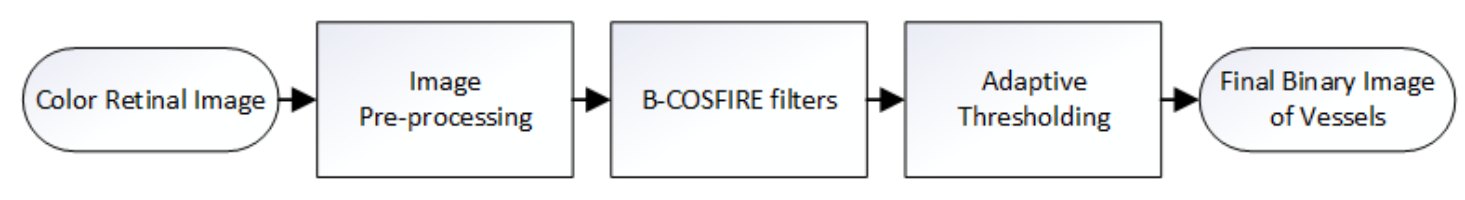

Figure 1. Overview of proposed method

\subsection{Pre-processing}

Green channel image displays the best contrast between retinal vessels and the retinal background [1] compared to either red or blue channels in an RI, thus it is extracted in the first step of preprocessing. Secondly, the contrast around the bright retinal region in the Field of View (FOV) against the dark background is smoothen using Soares's method [5] which involves detecting the FOV boundary, and then dilating it by replacing every pixel value just outside the border with the mean value of the pixels in its 8-neighbourhood that are inside the FOV. This step is repeated for 20 times and then, the dilated image is 
contrast-adjusted using contrast-limited adaptive histogram equalization (CLAHE) algorithm [21]. The resulting image will be the input to the next step, B-COSFIRE filter.

\subsection{B-COSFIRE}

B-COSFIRE is a novel bio-inspired filter that can be used to learn line patterns from an image signal [4]. It is a trainable filter in the sense that the filter's selectivity can be specified by providing a prototype pattern for the filter to detect. For application in RBV segmentation, a bar structure is used as the prototype pattern to configure the filter's selectivity towards vessel structures which are bar-shaped. The filter configuration involves the convolution of Difference of Gaussian (DoG) filter with the synthetic bar pattern in the prototype, followed by analyzing local maximum DoG responses along a specified number of concentric circles. Blurred and shifted responses of the filter are also considered to allow for some tolerance to preferred position of the prototype pattern. A DoG function can be represented by DoG(x,y) in equation 1 [19].

$$
D_{o} G_{\sigma}(x, y)=\frac{1}{2 \pi \sigma^{2}} \exp \left(\frac{-\left(x^{2}+y^{2}\right)}{2 \sigma^{2}}\right)-\frac{1}{2 \pi(0.5 \sigma)^{2}} \exp \left(\frac{-\left(x^{2}+y^{2}\right)}{2(0.5 \sigma)^{2}}\right)
$$

where $(x, y)$ is the pixel position in an image $I$, and $\sigma$ is the standard deviation of the Gaussian function that determines the extent of the surround. The inner Gausssian function of the DoG has the value $0.5 \sigma$ used as it's standard deviation value, i.e. half of standard deviation for outer Gaussian function. The response of a DoG filter defined in equation 1 to an image $I$ with intensity distribution $I\left(x^{\prime}, y^{\prime}\right)$ denoted as $C_{\sigma}(x, y)$ is computed by convolution:

$$
C_{\sigma}(x, y)=\left|I * D o G_{\sigma}\right|^{+}
$$

where $|.|^{+}$denotes half-wave rectification operation that changes all negative values to zeroes.

A center-on DoG filter is applied to the given prototype pattern to configure the B-COSFIRE filter. A center point, also known as the center of support of the B-COSFIRE filter, is selected and the DoG filter responses $c_{\sigma}(x, y)$ along several $\mathrm{k}$ concentric circles around the center point are considered. The dominant intensity variations around the pattern of interest is characterized by significant local maxima positions on the concentric circles. These points $\mathrm{i}$ are described by a tuple of three parameters $\left(\sigma_{i}, \rho_{i}, \phi_{i}\right)$ where $\sigma_{-} \mathrm{i}$ is the standard deviation of the DoG filter with the strongest response, while $\left(\rho_{i}, \phi_{i}\right)$ are the polar coordinates with respect to the center point. These set of 3 -tuples are denoted by $S=\left\{\left(\sigma_{i}, \rho_{i}, \phi_{i}\right) \mid i=1, \ldots, n\right\}$ with $\mathrm{n}$ representing the number of DoG responses considered. The DoG responses at the determined positions are used to calculate the B-COSFIRE output. The responses are first blurred to allow for some position tolerance of the points. Blurring is performed by computing the maximum value of weighted thresholded DoG responses whereas weighting is achieved by multiplying the responses of DoG filter with the coefficients of a Gaussian function whose standard deviation $\sigma$ ' is a linear function of the distance $\rho \_i$ from the filter's support center

$$
\sigma^{\prime}=\sigma_{0}^{\prime}+\alpha \rho_{i}
$$

where both $\sigma_{0}^{\prime}$ and $\alpha$ are constants. Next, each blurred DoG response is shifted by a distance $\rho_{-}$i going towards the opposite $\phi_{i}$ direction, meeting at the support center. The shift vectors are $\Delta x_{i}=-\rho_{i} \cos \phi_{i}$ and $\Delta y_{i}=-\rho_{i} \sin \phi_{i}$. The ith blurred and shifted response of the DoG filter is denoted by

$$
s_{\sigma_{i}, \rho_{i}, \phi_{i}}(x, y)=\max _{x^{\prime}, y^{\prime}}\left\{c_{\sigma_{i}}\left(x-\Delta x_{i}-x^{\prime}, y-\Delta y_{i}-y^{\prime}\right) G_{\sigma}\left(x^{\prime}, y^{\prime}\right)\right\}
$$

where $-3 \sigma^{\prime}<x^{\prime}, y^{\prime}<3 \sigma^{\prime}$. The output of a B-COSFIRE filter is then defined as the weighted geometric mean of all the blurred and shifted DoG responses corresponding to the set of tuples $S$ :

$$
r_{S}(x, y)=\left|\left(\prod_{i=1}^{|S|}\left(s_{\sigma_{i}, \rho_{i}, \phi_{i}}(x, y)\right)^{\omega_{i}}\right)^{1 / \sum_{i=1}^{|S|} \omega_{i}}\right|_{t}, \omega_{i}=\exp ^{\frac{\rho_{i}^{2}}{2 \sigma^{2}}}, \sigma=\frac{1}{3} \max _{i \in\{1 \ldots|S|\}}\left\{\rho_{i}\right\}
$$

where $|.|_{t}$ denotes thresholding the B-COSFIRE filter response at $t$ value $0 \leq t \leq 1$ of the maximum response. At this point, the filter is selective to the shape and orientation of the bar specified in the prototype pattern. To configure for selectivity to the same pattern at different orientations, the original orientation of the 
bar in the prototype pattern can be rotated at regular intervals to obtain a new set of 3 tuples $R_{\psi}(S)$ with different orientations $\psi$ :

$$
R_{\psi}(S)=\left\{\left.\left(\sigma_{i}, \rho_{i}, \phi_{i}+\psi\right)\right|^{\forall}\left(\sigma_{i}, \rho_{i}, \phi_{i}\right) \in \mathrm{S}\right\}
$$

With filters' selectivity now configured for multiple orientations, the responses of B-COSFIRE filters with different orientation are merged and the maximum value at every location $(x, y)$ are taken to produce the overall response:

$$
\widehat{r}_{S}(x, y)=\max _{\psi \in \Psi}\left\{r_{R_{\psi}(S)}(x, y)\right\}
$$

where $\Psi$ is a set of $n_{r}$ regular interval orientations defined as $\Psi=\left\{\frac{\pi}{n_{r}} i \mid 0 \leq i \leq n_{r}\right\}$. To detect vessel endings, a new B-COSFIRE filter with a prototype that depicts the vessel endings, i.e. only the upper half portion of the bar prototype in the previous filter, is configured. This vessel ending filter is referred to as asymmetric filter and the previous filter as symmetric filter. The final response is obtained by adding the responses of both symmetric and asymmetric filters before it is rescaled back to the original grayscale levels.

In this work, the published B-COSFIRE parameters for DRIVE and STARE dataset by the original authors in [19] were used. The original B-COSFIRE method specified a thresholding parameter to be determined using grid search for each specific dataset to binarize the output of the method as per Equation 5. In this study, two AT methods were investigated to automatically determine the optimal threshold $t$ which will eliminate the needs for (i) ground truth data and (ii) iterative search for optimal segmentation threshold for new datasets. Figure 2 show samples of retinal images from DRIVE and STARE database with their corresponding pre-processed images and B-COSFIRE filter responses.

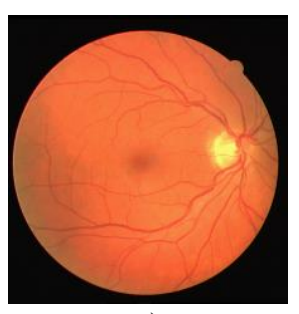

a)

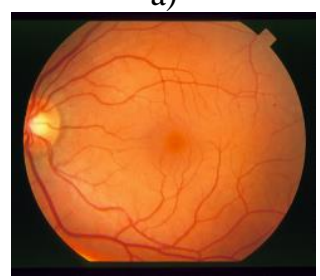

d)

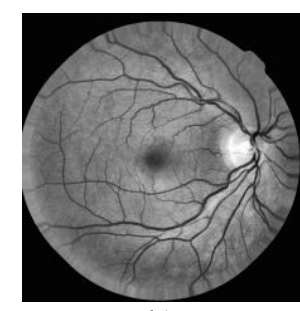

b)

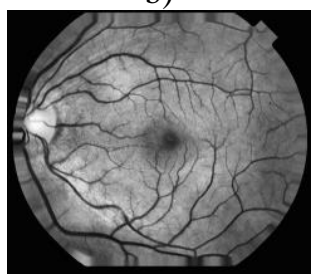

e)

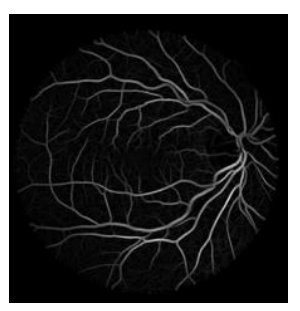

c)

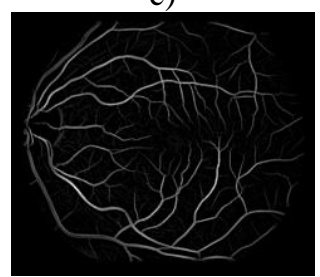

f)

Figure 2. (a), (d) Sample retinal images with their corresponding (b), (e) pre-processed images and (c), (f) B-COSFIRE responses for DRIVE (Row 1) and STARE (Row 2)

\subsection{Adaptive Thresholding (AT)}

We investigated two AT methods to determine the optimal threshold value for binarizing the B-COSFIRE method's output. First is the Otsu's method, which is widely used for segmentation of region of interests in an image [22]. The method assumes that every image consists of only two classes of pixels, namely the background pixels and the foreground pixels. To perform image segmentation into the two classes, the method chooses a threshold value based on the variance of the two classes. The other AT algorithm considered is ISODATA method [23]. This method relies on the assumption that the optimal threshold for image binarization is the average of the mean of of the two classes. ISODATA is an iterative method that starts with using the mean image for initialization to calculate the initial threshold, since the mean of the two classes are not initially known. The initial threshold will then be used to determine the next threshold value in search for the optimal value. The step is repeated until there are no significant changes in 
the threshold value or until a certain number of iterations is completed. These two methods were applied to the gray image output of B-COSFIRE filters $\widehat{r}_{S}(x, y)$ used to determine the threshold $t$ in Equation 5. The final output is the binary image resulting from thresholding the B-COSFIRE filters responses using the threshold $t$.

\subsection{Performance Measurement}

To quantify the segmentation performance, total number of true positive (TP), false positive (FP), true negative $(\mathrm{TN})$ and false negative (FN) pixels are calculated for each binary output image by comparing it to the manually segmented image. Using these values, a number of metrics normally used for segmentation performance measurement are then calculated. To compare the performance of the proposed method to the original B-COSFIRE method, the Accuracy (Acc), Sensitivity (Sn), Specificity (Sp), and Matthew's Correlation Coefficient (MCC) as defined below are calculated:

$$
\begin{aligned}
& A C C=\frac{T P+T N}{N}, S n=\frac{T P}{T P+F N}, S p=\frac{T N}{T N+F P}, \\
& M C C=\frac{(T P / N)-T P N \times T P P}{\sqrt{T P N \times T P P \times(1-T P N) \times(1-T P P)}}
\end{aligned}
$$

where $N=T N+T P+F N+F P, T P N=\frac{T P+F N}{N}$ and $T P P=\frac{T P+F P}{N}$. Sensitivity measures the ability of the method to successfully detect vessel pixels while specificity measures the ability to detect non-vessel pixels. MCC is a metric to normally measure performance of binary classifiers in the cases where the two classes are not balanced, like in this case with RBV segmentation where the number of vessel pixels is far less than the number of non-vessel pixels. In addition to these 4 metrics, another metric called G-mean proposed in [24] is calculated and defined as:

$$
G-\operatorname{mean}=\sqrt{S n \times S p}
$$

G-mean value of 1 indicates perfect segmentation while 0 indicates totally wrong prediction. It is a good choice of measure that incorporates both Sensitivity and Specificity into a single measure.

\section{RESULTS AND ANALYSIS}

For validation, the proposed method was used to segment retinal images from two publicly available retinal image databases, namely DRIVE [25] and STARE [26]. 20 images from the test set for DRIVE database and all 20 images in STARE database were used. Table 1 summarises the segmentation performance of the proposed method compared to original B-COSFIRE published in [19].

Table 1 . Performance results of the proposed B-COSFIRE with AT compared to original B-COSFIRE

\begin{tabular}{ccccccc}
\hline Database & Method & Acc & Sn & Sp & MCC & G-mean \\
\hline \multirow{3}{*}{ DRIVE } & Original B-COSFIRE & 0.9442 & 0.7655 & 0.9704 & 0.7475 & 0.8619 \\
& B-COSFIRE \& Otsu & 0.9450 & 0.6743 & 0.9851 & 0.7362 & 0.8150 \\
& B-COSFIRE \& ISODATA & 0.9456 & 0.6939 & 0.9829 & 0.7413 & 0.8259 \\
& Original B-COSFIRE & 0.9467 & 0.7716 & 0.9701 & 0.7335 & 0.8652 \\
& B-COSFIRE \& Otsu & 0.9511 & 0.7093 & 0.9791 & 0.7262 & 0.8334 \\
& B-COSFIRE \& ISODATA & 0.9507 & 0.7245 & 0.9769 & 0.7284 & 0.8413 \\
\hline
\end{tabular}

Comparing Otsu and ISODATA across the metrics in Table 1, it seemed that ISODATA outperformed Otsu except for the Specificity value. Thus, it is fair to conclude that ISODATA works better than Otsu in determining the threshold value for binarizing the B-COSFIRE filter output. It can also be seen from the table that using AT resulted in increase of Accuracy and Specificity values for both DRIVE and STARE database. However, for both databases, Sensitivity value which represent the method's ability to detect vessel pixels decreases, when using Otsu or ISODATA for thresholding. This is because both AT methods provide threshold values that are slightly higher than the optimized threshold determined using grid search method in original B-COSFIRE. This translates to lesser true positives segmented by ISODATA thresholding for most images, as illustrated in Figure 3(c), (g) and (h) where the output from AT method using ISODATA have detected vessels that are narrower in width and with lesser small vessels compared to 
the output obtained with optimized threshold in Figure 3(a), (e) and (f), respectively. However, there are also cases where ISODATA thresholding, as shown in Figure 3(d), detects more true positive pixels compared to optimized threshold shown in Figure 3(b).

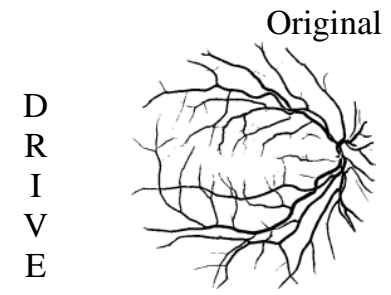

(a)

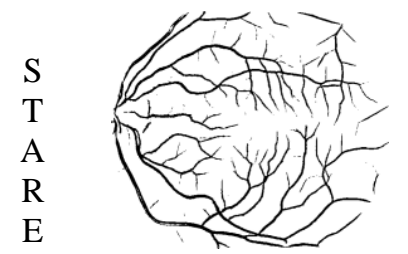

(e)

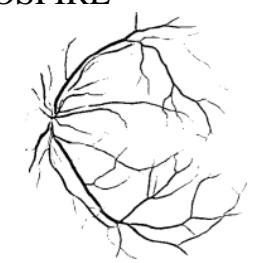

(b)

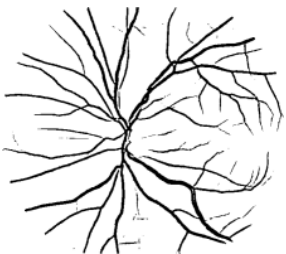

(f)

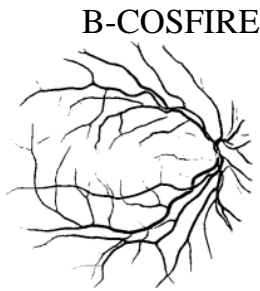

(c)

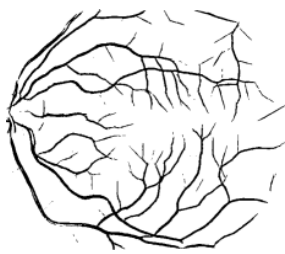

(g)

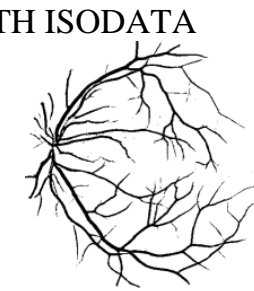

(d)

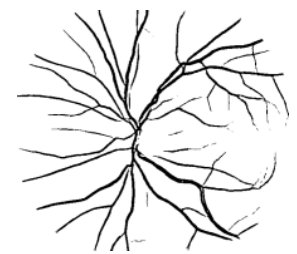

(h)

Figure 3. Sample vessel output images (a), (b), (e), (f) using original B-COSFIRE and (c), (d), (g), (h) using B-COSFIRE with ISODATA from the DRIVE database (top row) and STARE database (bottom row)

To increase the sensitivity value for ISODATA thresholding method, the threshold value determined by the AT method was reduced by $20 \%$, a value determined empirically. Qualitative comparison of the output between original ISODATA threshold and the reduced ISODATA threshold is shown in Figure 4. It can be seen that with decreased threshold value, more vessel pixels are detected in the form of enlarged main vessels and detection of additional smaller vessels. The improvement is also evident in the quantitative results summarised in Table 2. By using the decreased threshold value, as predicted the sensitivity values for both DRIVE and STARE databases increased along with an increase in G-mean values. When compared to the original B-COSFIRE results, the results obtained with decreased threshold value achieved better performance across three performance metrics namely Accuracy, Sensitivity and G-mean. However, the method's performance in detecting non-vessel pixels decrease slightly compared to original B-COSFIRE which could be due to slight increase in the number of false positive pixels.

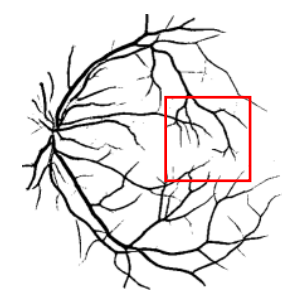

a)

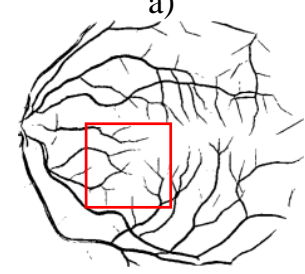

e)

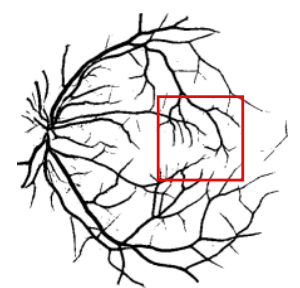

b)

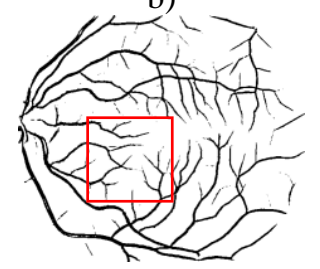

f)

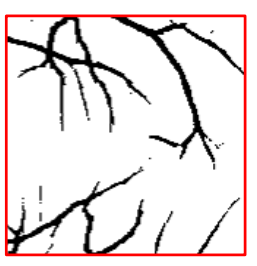

c)

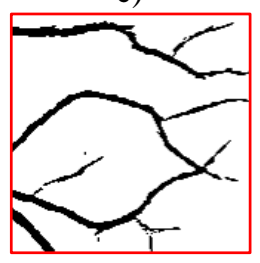

g)

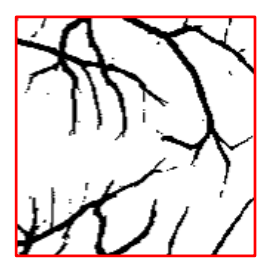

d)

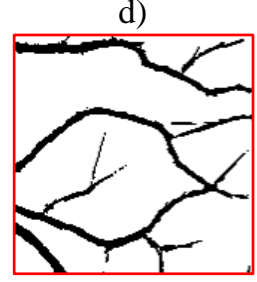

h)

Figure 4. Comparison of vessel outputs using (a), (e) ISODATA threshold, (b), (f) reduced ISODATA threshold for DRIVE (Row 1) and STARE (Row 2) databases. (c), (d), (g) and (h) are the enlarged red boxes in (a), (b), (e) and (f) respectively 
Table 2. Performance results of the proposed B-COSFIRE with Reduced Threshold (B-COSFIRE + ISODATA $(\mathrm{R})$ ) compared to original B-COSFIRE and other published methods

\begin{tabular}{|c|c|c|c|c|c|c|c|c|}
\hline \multirow{2}{*}{ Method } & \multicolumn{4}{|c|}{ DRIVE } & \multicolumn{4}{|c|}{ STARE } \\
\hline & Acc & $\mathrm{Sn}$ & $\mathrm{Sp}$ & $\mathrm{G}$ & Acc & $\mathrm{Sn}$ & $\mathrm{Sp}$ & $\mathrm{G}$ \\
\hline \multicolumn{9}{|l|}{ Unsupervised } \\
\hline Original B-COSFIRE [19] & 0.9442 & 0.7655 & 0.9704 & 0.8619 & 0.9467 & 0.7716 & 0.9701 & 0.8652 \\
\hline B-COSFIRE \& ISODATA & 0.9456 & 0.6939 & 0.9829 & 0.8259 & 0.9507 & 0.7245 & 0.9769 & 0.8413 \\
\hline B-COSFIRE \& ISODATA (R) & 0.9446 & 0.7818 & 0.9688 & 0.8703 & 0.9471 & 0.7957 & 0.9648 & 0.8762 \\
\hline Mendonca [8] & 0.9463 & 0.7344 & 0.9764 & 0.8468 & 0.9479 & 0.6996 & 0.9730 & 0.8251 \\
\hline Fraz [9] & 0.9430 & 0.7152 & 0.9768 & 0.8358 & 0.9442 & 0.7311 & 0.9680 & 0.8413 \\
\hline Martinez-Perez [12] & 0.9344 & 0.7246 & 0.9655 & 0.8364 & 0.9410 & 0.7506 & 0.9569 & 0.8475 \\
\hline \multicolumn{9}{|l|}{ Supervised } \\
\hline Asl [13] & & 0.7428 & 0.9732 & 0.8502 & & 0.7419 & 0.9706 & 0.8486 \\
\hline Orlando [18] & & 0.7897 & 0.9684 & 0.8745 & & 0.7680 & 0.9738 & 0.8648 \\
\hline Marin [14] & 0.9452 & 0.7067 & 0.9801 & 0.8322 & 0.9526 & 0.6944 & 0.9819 & 0.8257 \\
\hline Soares [5] & 0.9466 & 0.7332 & 0.9782 & 0.8469 & 0.9480 & 0.7207 & 0.9747 & 0.8381 \\
\hline Fraz [16] & 0.9480 & 0.7406 & 0.9807 & 0.8522 & 0.9534 & 0.7548 & 0.9763 & 0.8584 \\
\hline Strisciuglio [15] & 0.9454 & 0.7777 & 0.9702 & 0.8686 & 0.9534 & 0.8046 & 0.9710 & 0.8839 \\
\hline
\end{tabular}

Result of the proposed B-COSFIRE \& ISODATA (R) method (with Acc=0.9446) compared to other published methods show comparable performance. It also has the highest Sensitivity and G-mean values of 0.7818 and 0.8703 for DRIVE, and 0.7957 and 0.8762 for STARE compared to other unsupervised methods. For supervised methods, only Orlando's method for DRIVE and Strisciuglio's method for STARE have slightly higher Sensitivity and G-mean values than the proposed method. Highest Specificity value for DRIVE was achieved by B-COSFIRE+ISODATA method at 0.9829 while for STARE by Marin's method at 0.9819 .

\section{CONCLUSION}

In this paper, an efficient unsupervised method to segment RBVs on retinal image is proposed by combining trainable B-COSFIRE filter with AT. Original B-COSFIRE implementation used grid search to determine the threshold value that maximizes the MCC value. Two AT methods were investigated for binarizing B-COSFIRE filter responses, namely ISODATA and Otsu. Results indicate that ISODATA achieved better performance across most of the considered metrics compared to Otsu. While the combined BCOSFIRE and ISODATA method achieved better Accuracy and Specificity values compared to the original B-COSFIRE, Sensitivity, MCC and G-mean values are notably lower. To improve the overall performance, the threshold determined by ISODATA method was reduced by $20 \%$. This resulted in significant increase in Sensitivity and G-mean values, which are directly related to the ability of the proposed method to better detect vessel pixels. Comparison of the results obtained to other published methods indicated comparable performance to both supervised and unsupervised methods, with average processing time of around 5 seconds per image. The use of AT eliminates the need for availability of ground truth data for finding the optimal threshold value using grid search.

\section{ACKNOWLEDGEMENTS} 2015-012).

This research is supported by MOHE Malaysia (FRGS/1/2015/TK04/UKM/01/3) and UKM (DIP-

\section{REFERENCES}

[1] Fraz M. M., et al., "Blood vessel segmentation methodologies in retinal images - A survey," Comput Methods Programs Biomed, vol. 108, pp. 407-33, 2012.

[2] Almotiri J., et al., "Retinal Vessels Segmentation Techniques and Algorithms: A Survey," Appl Sci., vol. 8, pp. $155,2018$.

[3] Chaudhuri S., et al., "Detection of blood vessels in retinal images using two-dimensional matched filters," IEEE Trans Med Imaging, vol. 8, pp. 263-9, 1989.

[4] Strisciuglio N. and Petkov N., "Delineation of line patterns in images using B-COSFIRE filters," 2017 Int. Conf. Work. Bioinspired Intell., IEEE, pp. 1-6, 2017.

[5] Soares J. V. B., et al., "Retinal vessel segmentation using the 2-D Gabor wavelet and supervised classification," Med Imaging, IEEE Trans, vol. 25, pp. 1214-22, 2006.

[6] Bekkers E., et al., "A multi-orientation analysis approach to retinal vessel tracking," J Math Imaging Vis., vol. 49, pp. 583-610, 2014. 
[7] Chutatape O., et al., "Retinal blood vessel detection and tracking by matched Gaussian and Kalman filters," Proc 20th Annu Int Conf IEEE Eng Med Biol Soc Vol20 Biomed Eng Towar Year 2000 Beyond (Cat No98CH36286), vol. 6, pp. 44-9, 1998.

[8] Mendonça A. M. and Campilho A., "Segmentation of retinal blood vessels by combining the detection of centerlines and morphological reconstruction," IEEE Trans Med Imaging, vol. 25, pp. 1200-13, 2006.

[9] Fraz M. M., et al., "An approach to localize the retinal blood vessels using bit planes and centerline detection," Comput Methods Programs Biomed., vol. 108, pp. 600-16, 2012.

[10] Nguyen U. T. V., et al., "An effective retinal blood vessel segmentation method using multi-scale line detection," Pattern Recognit., vol. 46, pp. 703-15, 2013.

[11] Strisciuglio N., et al., "Multiscale Blood Vessel Delineation Using B-COSFIRE Filters, Springer, Cham," pp. 300$12,2015$.

[12] Martinez-Perez M. E., et al., "Segmentation of blood vessels from red-free and fluorescein retinal images," Med Image Anal, vol. 11, pp. 47-61, 2007.

[13] Asl M. E., et al., "Tracking and diameter estimation of retinal vessels using Gaussian process and Radon transform," J Med Imaging, vol. 4, pp. 1, 2017.

[14] Marín D., et al., "A New Supervised Method for Blood Vessel Segmentation in Retinal Images by Using GrayLevel and Moment Invariants-Based Features," IEEE Trans Med Imaging, vol. 30, pp. 146-58, 2011.

[15] Strisciuglio N., et al., "Supervised vessel delineation in retinal fundus images with the automatic selection of B COSFIRE filters," Mach Vis Appl., 2016.

[16] Fraz M. M., et al., "An ensemble classification-based approach applied to retinal blood vessel segmentation," IEEE Trans Biomed Eng., vol. 59, pp. 2538-48, 2012.

[17] Vermeer K. A., et al., "A model based method for retinal blood vessel detection," Comput Biol Med., vol. 34, pp. 209-19, 2004.

[18] Orlando J. I., et al., "A Discriminatively Trained Fully Connected Conditional Random Field Model for Blood Vessel Segmentation in Fundus Images," IEEE Trans Biomed Eng., vol. 64, pp. 16-27, 2017.

[19] Azzopardi G., et al., "Trainable COSFIRE filters for vessel delineation with application to retinal images," Med Image Anal., vol. 19, pp. 46-57, 2015.

[20] Vostatek P., et al., "Performance comparison of publicly available retinal blood vessel segmentation methods," Comput Med Imaging Graph, 2016.

[21] Pizer S. M., et al., "Adaptive histogram equalization and its variations" Comp Vision, Graph Image Process, vol. 39, pp. 355-68, 1987.

[22] Otsu N., "A Threshold Selection Mthd from Gray-Level Histograms," IEEE Trans Syst Man Cybern, vol. C, pp. 62-6, 1979.

[23] Ball G. H. and Hall D. J., "ISODATA, a novel method of data analysis and pattern classification," Analysis, pp. 179,1965

[24] Orlando J. I., et al., "Retinal blood vessel segmentation in high resolution fundus photographs using automated feature parameter estimation" in Brieva J., et al., 13th Int. Conf. Med. Inf. Process. Anal., vol. 10572, pp. 8, 2017.

[25] Staal J., et al., "Ridge-based vessel segmentation in color images of the retina," IEEE Trans Med Imaging, vol. 23, pp. 501-9, 2004.

[26] Hoover A. and Goldbaum M., "Locating the optic nerve in a retinal image using the fuzzy convergence of the blood vessels," IEEE Trans Med Imaging, vol. 22, pp. 951-8, 2003.

\section{BIOGRAPHIES OF AUTHORS}

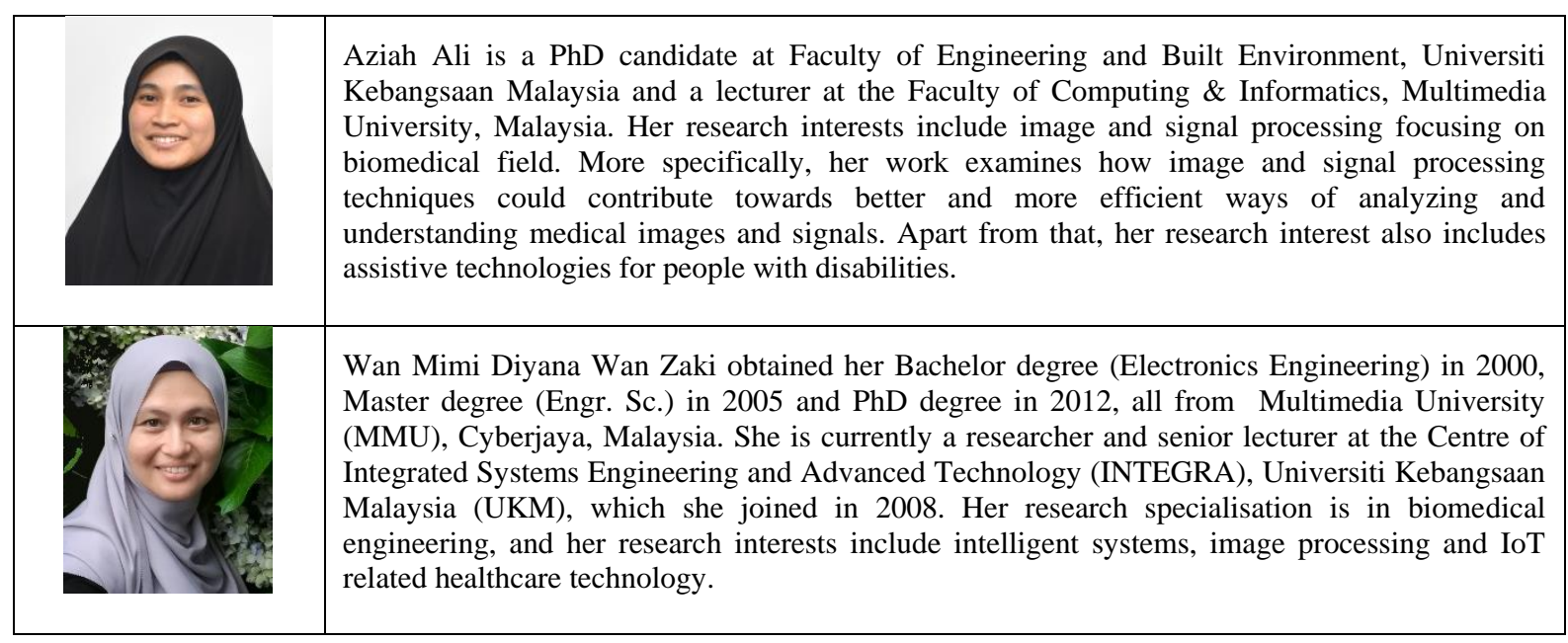

Indonesian J Elec Eng \& Comp Sci, Vol. 13, No. 3, March 2019 : 1199 - 1207 


Aini Hussain obtained her B.Sc. in Electrical Engineering from Louisiana State University
(LSU), Baton Rouge, USA; M.Sc. in Systems and Control from the University of Manchester
Institute of Science and Technology (UMIST), Manchester, U.K., and Ph.D. in Electrical and
Electronic Engineering from the National University of Malaysia in 1985, 1991 and 1997,
respectively. She is a Professor and currently, the Chair of the INTEGRA research center also
known as "Centre for Integrated Systems Engineering and Advanced Technologies" Her
research, for which she has received funding, focuses on Intelligent Systems and Image
Processing. Her current research interests are in machine learning, pattern recognition and video
\& image processing.

